\title{
TMJ Arthritis
}

Subhashini Ramasubbu ${ }^{1}$, Shivangi Gaur², Abdul Wahab P.U. ${ }^{3}$, Madhulaxmi Marimuthu ${ }^{4}$

1, 2, 3, 4 Department of Oral and Maxillofacial Surgery, Saveetha Dental College and Hospitals, Saveetha Institute of Medical and Technical Sciences, Saveetha University, Velappanchavadi, Chennai, Tamil Nadu, India.

\section{ABSTRACT}

\section{BACKGROUND}

Temporomandibular Joint (TMJ) arthritis affects the joint and the surrounding musculature. Like any other joints, causes of temporomandibular joint arthritis could be rheumatoid arthritis, osteo arthritis, or psoriatic arthritis. Severity of the disease differs from each other ranging from mild to severe. In case of temporomandibular joint trauma, it may lead to degeneration of the joint which may result in ankylosis of the joint if it is left untreated. In case of inflammatory arthropathies, even after the treatment, inflammation of the joint still persists. To suppress the inflammation, patients can be prescribed immunosuppressive treatment. Long term use of immunosuppressants is deleterious and may lead to failure of organs. One more adverse effect of immunosuppressive drugs is that it makes the patient prone for infections if the patient undergoes surgery. Symptoms of temporomandibular joint arthritis include pain on involved side, restricted mouth opening, and difficulty in eating. Origin of pain may be from the joint itself or from the muscles attached to it or from both. The patient will complain of mild to severe pain. The objective of this study is to assess TMJ arthritis in those with above said inflammatory arthropathies. This article is to highlight the peculiarities of TMJ arthritis secondary to those inflammatory arthropathies and how to best manage these ailments, which should guide when referral to a specialist TMJ surgeon is appropriate. The aim of this review is to discuss about the various causes of TMJ arthritis, etiopathogenesis, clinical features, investigations and the management of temporomandibular joint Arthritis.

\section{KEY WORDS}

Temporomandibular Joint Arthritis, Rheumatoid Arthritis, Psoriatic Arthritis, Osteoarthritis, Ankylosing Spondylitis
Corresponding Author:

Dr. Subhashini Ramasubbu.

Department of Oral and Maxillofacial Surgery, Saveetha

Dental College and Hospitals, Saveetha Institute of Medical and Technical Sciences, Saveetha University, No 162, Poonamallee High Road, Velappanchavadi, Chennai, Tamil Nadu, India.

E-mail: meetsubha07@gmail.com

DOI: $10.14260 /$ jemds/2020/690

How to Cite This Article:

Ramasubbu S, Gaur S, Abdul Wahab PU, et al. TMJ arthritis. J Evolution Med Dent Sci 2020;9(42):3152-3158, DOI: $10.14260 /$ jemds/2020/690

Submission 18-07-2020,

Peer Review 12-09-2020,

Acceptance 18-09-2020,

Published 19-10-2020.

Copyright (C) 2020 Subhashini Ramasubbu et al. This is an open access article distributed under Creative Commons Attribution License [Attribution 4.0 International (CC BY 4.0)] 


\section{BACKGROUND}

Temporomandibular Joint arthritis affects the joint and the surrounding musculature. Like any other joint, causes of temporomandibular joint arthritis could be rheumatoid arthritis, osteo arthritis or psoriatic arthritis. The aim of this review is to discuss about the various causes of TMJ arthritis, aetiopathogenesis, clinical features, investigations and the management of temporomandibular joint arthritis.

\section{RHEUMATOID ARTHRITIS}

Rheumatoid arthritis is an autoimmune, inflammatory disorder causing inflammation in and around the joint, resulting in deformity and loss of function ${ }^{1}$. It is a chronic painful condition involving almost all joints, causing erosion of the joints and the presentation is usually symmetric. It usually involves peripheral joints. Involvement of TMJ is rare and it is the last joint to get involved by the disease. Rheumatoid arthritis is prevalent worldwide with a percentage of 1 percent. Women are most commonly affected than men with a ratio of $3: 1$. The age distribution is between 35 and 50 years. Percentage of involvement of TMJ is $2-88$ $\% .^{2-4}$ But majority of studies shows TMJ involvement in $50 \%$ of rheumatoid arthritis patients ${ }^{5-7}$ If it involves the joint, pain is the major problem, later it leads to inflammation, which in turn causes restricted movement, stiffness of the joint and muscle spasm. ${ }^{8}$

\section{Aetiopathogenesis}

Aetiology and pathogenesis of the disease are multifactorial. It involves macrophages, $\mathrm{T}$ cells, $\mathrm{B}$ cells, chondrocytes, fibroblasts and dendritic cell. Combined genetic and immunological factors are associated with the disease. There are genes and epigenetic modification in the development of disease. Environmental factors like cigarette smoking, pollutants like silica, some bacterial and viral infection are also equally important. In the initial stage, there are disturbance in protein modification, followed by APC (Antigen Presenting Cell) and Fibroblast-Like Synoviocytes (FLS) activation. Very rarely trauma to the joint or any acute injury mat trigger the disease.

\section{Classification of Rheumatoid Arthritis Based on Radiographic Changes \\ Classification is based on radiographic changes. Larsen classified into 6 grades \\ 1. Normal \\ 2. Slight narrowing of joint space \\ 3. Early abnormality, slight narrowing of joint space, slight erosion \\ 4. Moderate disturbance, joint space eroded and reduced \\ 5. Destruction is severe. Eroded, reduced joint space, deformity is seen \\ 6. Mutilating, disappearance of joint space, bony deformity}

\section{General Symptoms of Rheumatoid Arthritis}

1. Swollen Joint

2. Pain

J Evolution Med Dent Sci / eISSN - 2278-4802, pISSN - 2278-4748 / Vol. 9 / Issue 42 / Oct. 19, 2020
3. Restricted movement

4. Fatigue

5. Fever

6. Loss of weight

7. Mild joint discomfort to severe pain

8. In chronic stage, it can cause cartilage loss, erosion and generalized weakness.

9. Deformity

10. Destruction of joint

11. Loss of function

\section{TMJ Symptoms}

1. Pain in preauricular region

2. Swelling

3. Crepitus

4. Impairment of the function

5. Occlusal derangement

6. Sometimes in advanced stage, they may be anterior open bite

7. Stiffness of the joint

8. In children, it may affect growth of the mandible, eventually leads to facial deformity and ankylosis with occlusal deformity. Patient is unaware of the relationship between TMJ and arthritis ${ }^{4,9}$

\section{Diagnosis}

Diagnosis is based on patient's clinical history, clinical findings, radiographs, blood investigations. Multidisciplinary approach is needed. ${ }^{10}$

\section{Investigations}

1. Routine blood investigation

2. ESR, CRP

3. Rheumatoid factor

4. ANA is a frequent finding in RA patients

5. Citrulline Antibody tests (done in dreadful cases)

6. $\mathrm{OPG}$

7. MRI

8. CT

9. TMJ Tomogram

10. Arthrography

11. СBCT

Regarding rheumatoid arthritis, there won't be any changes in early stage of disease. The findings may vary from $19-86 \%$. In chronic stage or in severe stage there will be flattening of condylar head, erosion of the joint, reduced joint space, spiked or pencil head deformity of condyle and in some cases sub cortical cysts.

\section{Management}

Medical Therapy - Aim of the therapy is to relieve pain. It can resolve symptoms in $80 \%$ of the patients. ${ }^{11}$ Therapy includes reassurance, soft diet, physiotherapy, NSAID's ${ }^{12} \&$ occlusal splints. Effective management includes DMARD's (Disease Modifying Anti-Rheumatoid Drugs). They are second line of drugs. They are methotrexate and HCQ. These drugs prevent further progression of the disease. Newer DMARD's are Leflunomide, Etanercept, Infliximab, Rituximab and 
Abatacept. Though physiotherapy has no long-term benefits, it is not harmful. Topical NSAID's (Nonsteroidal AntiInflammatory Drug) is as effective as oral preparation and should be taken 4 times a day for 4 weeks and it has fewer side effects. ${ }^{13-15}$ Low dose tricyclic antidepressants can be given for myofascial pain. ${ }^{16}$ Occlusal splints should be worn at night. It permits masticatory muscle rest, reduces myofascial pain, it reduces load on TMJ as well. ${ }^{17}$ Benefits of DMARD's are, TMJ is treated simultaneously with other joints by suppression of inflammation. ${ }^{18-23}$ In the management of arthritis, the choice of NSAID remains confusing and controversial.

Local Anesthesia and Steroid Injection - Pain can be temporarily controlled by LA (lignocaine $1 \%$ or $2 \%$ ) infiltration into joint space. Site of injection is $10 \mathrm{~mm}$ along and $2 \mathrm{~mm}$ below the line joining tragus and lateral canthus of eye. Bupivacaine $0.5 \%$ can be injected for reducing myofascial pain and spasm. When it is injected in temporalis muscle, localized muscle paralysis occurs for up to 6 months and reduced pain, which was measured by VAS (Visual Analogue Scale) in $79 \%$ patients $^{24}$

Arthroscopy and Arthrocentesis - Minimally invasive, tolerated, cost effective carried out under LA. It will elucidate pathology, saline lavage of the joint under pressure, it flushes out debris that prevent normal movement. The procedure can be done to the upper joint space. For lower joint space, open operative procedure is needed. The Cochrane review found that arthroscopy has greater improvement in mouth opening after 1 year.

Open Joint Surgery - For lower joint space disease open surgery can be done. Synovectomy is a high-risk procedure, because of close proximity to trigeminal nerve branches, IJV (Internal Jugular Vein) and carotid vessels. It can be combined with discectomy. Eminoplasty, eminectomy or adhesiolysis can also be done. In previously unoperated joint, arthroscopy / arthrocentesis should be done first. ${ }^{25-27}$

TMJ Replacement Surgery - Patients with restricted mouth opening, prolapse, poorer prognosis require total joint replacement. Currently 2 systems are in use. First is patientfitted with total TMJ replacement, using CAD-CAM model of the joint form3D scan. Second system is the Biomet microfixation TMJ replacement. TMJ replacement surgery was first reported in 1990s with alloplastic material. Researches are focused on the assessment of longevity and the long-term functional results of the implants. It also focuses on patient's satisfaction. Thorough clinical and radiological investigations is needed for good results.

The surgical method of temporomandibular joint replacement is controversial. Already several techniques like autogenous and alloplastic material have been described in literature. There are some specific conditions like pathologies which need total joint replacement with alloplastic and these can be descried as absolute indication. Other conditions which require joint replacement are ankylosis or recurrent ankylosis, graft failure, inflammatory joint disease and other situation where the anatomy is destroyed. Relative indications are condylar fracture, AVN, tumours requiring resection, congenital anomalies like hemifacial microsomia.

The custom-made prosthesis is designed by computer aided systems, and it is designed using stereolithographic models. Stereolithographic models can be obtained through 3-D computed tomography.

Other Treatment Modes - In one study patient with RA (Rheumatoid Arthritis) who has anterior open bite, orthodontic approach was done to improve occlusal and articular condition, promoting functional mandibular adaptation, reconstructing occlusion, which leads to condylar adaptive remodeling. For assessing myogenous temporomandibular disorders, thermography is also used. ${ }^{28}$

\section{PSORIATIC ARTHRITIS}

Psoriatic arthritis is an inflammatory condition which presents in psoriasis patients even before the appearance of skin rashes. This inflammatory condition involves mostly the distal interphalangeal joints, knee joint, spine, elbow and ankle joint can also be involved. Psoriatic arthritis of temporomandibular joint is rare. First case of psoriatic arthritis of TMJ was reported in early $19^{\text {th }}$ century.

\section{Subgroups}

1. Symmetric polyarthritis

2. Asymmetric Oligo-arthritis

3. Arthritis of distal interphalangeal joints

4. Spondylitis

5. Destructive arthritis

\section{Aetiology}

It includes genetic, environmental factors and immunologic factors.

\section{Clinical Features}

1. Psoriatic rashes in buccal mucosa, lip, palate and gingiva

2. Buccal mucosa is commonly involved.

3. Gingiva ang palate are rarely involved

4. Lesion may be plaque-like ( $44 \%$ ), erythematous (24\%) or with mixed appearance (14\%)

5. Sometimes present as ulcerative lesion, vesicular or pustular lesion

6. Benign migratory glossitis, fissured tongue may be a feature in psoriatic arthritis.

\section{TMJ Psoriatic Arthritis}

Usually rare. Associated with long standing and severe psoriatic arthritis. Mostly erosive type. Signs and symptoms include

1. Clicking sound

2. Restricted jaw movements

3. Stiff joint

4. Tenderness

5. Pain in ears

6. Pain In masticatory muscles 
The disease progresses from arthralgia to fibrosis, then structural changes results in crepitus. Since the presentation is same as rheumatoid arthritis, it should be ruled out first. Boyle and Buchanan reported three things to confirm psoriatic arthritis. They are 1. Presence of psoriasis, 2. Polyarthritis of erosive type, 3. RA-Negative

\section{Investigations}

Linderg and Ericson first described radiographic findings, most common findings are

1) Erosion of the joint with osteoporosis (Osteolysis)

2) Osteophytes

3) Reduced joint space

4) Flattening of condylar head

5) Sclerosis

\section{Other Additional Investigations}

MRI - Effusion and for disc disorders

USG - Effusion of the joint

\section{Treatment}

The main aim of the treatment is to relieve tenderness or pain. Psychiatric counselling and reassurance. NSAIDs to relieve pain, steroids. Physiotherapy for the function of joint.

\section{OSTEOARTHRITIS (OA)}

A form of arthritis which is characterized by degeneration of the joint. It involves both hard and soft tissues. This can alter anatomical structure of the joint which leads to the alteration in peripheral and central pain pathways results in pain the most commonly affected joints are weight bearing joints such as knee, spine, hip and also fingers.

It can affect almost all joints of the body. Osteoarthritis of temporomandibular joint affects cartilage, bone, synovium and other associated hard and soft tissues which leads to remodeling, abrasion and deterioration of the joint. $\mathrm{OA}$ affects around $15 \%$ of the world population. It increases with increase in age. Age affected is $5^{\text {th }}$ and $6^{\text {th }}$ decade. Women are most commonly affected than men. The reason being estrogen receptor alpha polymorphin.

American Academy of Oro Facial Pain categorized TMJ OA as primary and secondary.

Primary - Absence of any local or systemic factors

Secondary - Has previous trauma or disease.

\section{Investigations}

1. Blood investigations to rule out rheumatoid arthritis

2. Panoramic X-Rays

3. MRI

4. CT

5. $\mathrm{CBCT}$

\section{Treatment}

After diagnosis, treatment depends on the stage, symptoms and also the comorbid condition. The main goal is to suppress the inflammation, to restore functions, to prevent deformity, and ultimately the pain. Only symptomatic treatment is required. The treatment can be non-surgical or surgical depending on the severity. Non-surgical treatment include physiotherapy, PES, pharmacological, steroids, hyaluronic acid injections. Early detection and treatment provide best results.

\section{Pharmacological Treatment}

It is based on cost, mode of administration, adverse effects, patient compliance. The treatment includes the use of acetaminophen, NSAIDS, COX-2 inhibitors, topical analgesics. Intraarticular injection of steroids, hyaluronic acid, glucosamine for pain relief. Glucosamines is used as disease modifying agent. Opioids can also be used for relieving refractory pain.

Acetaminophen is known as the oldest, most commonly used drug for the treatment of OA. In spite of its least effectiveness in controlling pain, it can be still used due to its least toxicity. So, it can be used as the first line of drugs. The maximum dose of $4 \mathrm{gm} /$ day can be given. It has liver toxicity so in case of alcoholics or existing liver problems it should be used with caution.

As already discussed previously, NSAIDs can be used for pain and to slow down the inflammatory process. Eventually it can slowdown degeneration as well. It has renal toxicity so it should be used with caution. And also, in the patients with gastrointestinal disturbances it should be used with caution.

Supplements like chondroitin sulphate, glucosamine can be given. A combination of glucosamine and NSAIDs known to reduce pain in some patients. These are basically disease modifying drugs. Vitamin D has controversial effect in these patients.

Topical NSAIDs can also be prescribed. Among all topical NSAIDs, diclofenac shown to be effective in reducing pain. Intra articular steroid injection, arthrocentesis can be done if the above said treatment failed to reduce the pain.

\section{Surgery}

Condylotomy, autogenous disc replacement and total joint replacement are some of the surgical procedures for osteoarthritis of TMJ.

\section{Ankylosing Spondylitis}

It is also called as Marie-Strumpell disease. It is also a chronic inflammatory disease which usually affects axial skeleton and it also affects peripheral joint. Unlike other autoimmune disorders which usually affects females, this disease affects male in the ratio of $2.4: 1$. Synovium, cartilages, the tendon attachment site, and ligament attachment site are the different parts of the joints which gets affected in ankylosing spondylitis.

Temporomandibular joint (TMJ) involvement in ankylosing spondylitis ranges from $4 \%-35 \%$. If the joint is involved there will be tenderness, limited mouth opening, difficulty in chewing, difficulty or inability to eat.

\section{Investigations}

1. Radiographs

2. CT 
3. Routine Blood investigations

4. ESR

5. CRP

6. Human Leucocyte Antigen B-27

7. MRI to rule out sacroiliitis

\section{Treatment}

After investigation, diagnosis was made. Treatment is a combination of conservative methods. Conservative methods include, rest, physiotherapy, medical treatment include Sulfasalazine $1 \mathrm{~g}$ BD daily and Tablet Indomethacin $50 \mathrm{mg}$ daily. If it is not subsided, then they can undergo biological therapy. Splint, prosthesis can also be advised.

\section{DISCUSSION}

RA is a chronic, systemic, progressive, auto immune disease characterized by bilateral arthritis, inflammation of synovial membrane. Females are affected three times more than males. In a study, it is confirmed with Ratio of 0.064 in favor of women. Clinical course of rheumatoid arthritis may vary from mild discomfort of joint to chronic polyarthritis, most importantly pain, joint deformity, and swelling.

Helenis et $\mathrm{al}^{2}$ reported that TMJ is the last joint to get involved, though it affects every joint. GD Kumaresan ${ }^{29}$ concluded that, in patients with mean duration of disease found to be 11.2 years while TMJ involvement was found to be 1.7 years. Yi-chun Lin et $\mathrm{al}^{4}$ surveyed TMD (TemporoMandibular Disorder) and RA in 56 patients. Among them $51.8 \%$ of them had TMJ symptoms and others were clinically silent. $91.1 \%$ of patients have subjective TMD. This is due to overshadowing of joint pain elsewhere in the body. The patients subjectively reduce TMJ motion by avoiding hard food and by talking less.

Compared to other joints, since TMJ has high vascularity in retrodiscal tissues, it acts as a drainage for exudates thereby reducing swelling and pain. Yi-chun Lin et $\mathrm{al}^{4}$ concluded that despite severe destruction of TMJ, $23.2 \%$ of patients had maximal mouth opening $<40 \mathrm{~mm}$ and limited mouth opening in only $14.9 \%$ of patients. Marbach ${ }^{30}$ stated that osteoarthritis is a common disease of TMJ. Gynther et al concluded that arthroscopic, histologic and immunohistochemical studies revealed that TMD disease in $\mathrm{OA}$ and RA is similar. Radiographic changes include cortical erosion, reduced joint space, sharpen pencil head / spiked deformity of condylar head.

Abhijeet Shirish reported that RA patients had erosion of condyle (85\%) then followed by condylar sclerosis. Yu-chun Lin et al found that majority of RA (29.6\%) had TMJ problem in 1 year and $51.8 \%$ developed more than 1 year. He also reported that one of the rare occurrences is ankylosis. It occurs only in the chronic stage. If it occurs, it will affect both the sides. In the severe cases retrognathia may be one common finding, there may be reduction in mandibular height, and in very severe cases there may be anterior open bite. In ankylosis, because of retrognathia there may be airway obstruction especially in supine position. But airway obstruction is intermittent.

Various conditions may affect the temporomandibular joint (TMJ), but its incidence in individual joint disorders is low. Particularly in inflammatory arthropathies like rheumatoid arthritis, psoriatic arthritis and ankylosing spondylitis, osteoarthritis appears to have a tendency to affect the joint.

Symptoms include tenderness, restricted mouth opening, locking, crepitus, which together can lead to significant impairment of the joint. Jaw rest, lifestyle modification, occlusal splint, and pharmacological therapy, including Disease-Modifying Antirheumatic Drugs (DMARDs) and simple analgesics (NSAIDs), are the initial treatment and will improve most symptoms in most patients.

If the disease progresses, there may be deterioration of symptoms. In those cases, where the symptoms are getting deteriorated, TMJ arthroscopy and arthrocentesis can help reducing the pain, improvement in mouth opening, and relieve locking. These minimally invasive procedures have certain complications and can be repeated. For the severe cases where pharmacological agents do not relieve the symptoms, the last resort is surgery. Surgeries are to be done to repair or to remove the damaged disc or to refine the anatomy of the joint. But surgeries are done in selective cases only.

Total temporomandibular joint replacement is reserved for patients where joint is deformed, or complete fusion has occurred or in whom other treatments have failed to provide adequate symptomatic control. It gives excellent outcomes and is approved by the National Institute of Health and Care Excellence (NICE), UK. Knowledge of the assessment, diagnosis and treatment of the TMJ, which differs from all other joints affected by inflammatory arthritis due to its unique anatomy and function, is not widespread outside of the field of oral and maxillofacial surgery. But again, it is technique sensitive and it needs skilled surgeon to do.

Zhou et al reported that, only $5 \%$ of TMJ involvement is there in psoriatic arthritis. ${ }^{31} \mathrm{He}$ also reported that it is not a common finding. The patients usually have pain and stiffness in neck and shoulders. And they also have muscle pain while chewing, locking of jaw is also some common symptoms in psoriatic arthritis of jaw. There will be clicking and crepitation also. Clicking is due to disc derangement. Crepitation may be due to structural changes of the joint, it increases with age. Dervis reported $16.1 \%$ of psoriatic arthritis patients had par functional habits. Most common habit is bruxism.

Aetiopathogenesis of osteoarthritis is complex and it is multifactorial. Genetics and age ate the risk factors. Other factors are previous trauma, repetitive loading, torsional load, long term micro trauma of the joint, disturbances of jaw musculature, instability of the joint, internal derangement of TMJ, discectomy, laxity of ligaments, systemic comorbidity like osteoarthritis, congenital abnormality, developmental disturbances.

The early damage of the cartilage may be due to mechanical factors or environmental factors. These factors initiate biochemical changes in the bone and the surrounding muscle and other soft tissues. This triggers the immune system which aggravate the inflammatory response, by means of various inflammatory mediators such as cytokines and chemokines. This process combined with complement system activation, which releases Matrix Metallo-Proteinase (MMP) and prostaglandins (PGE). These products degrade the cartilage, and leads to damage of articular cartilage. Eventually results in degradation of the joint and erosion. 
These changes initiate inflammatory response and it leads to remodelling of the bone.

\section{CONCLUSIONS}

Although TMJ is the last joint to get involved in all inflammatory joint diseases, it is important to know the clinical history. Based on the history, clinical findings, and radiographic studies, diagnosis is made. Though it is uncommon, when severe restriction occurs, it is debilitating. Early advice and management from oral and maxillofacial surgeon are necessary to slow down the progression of disease. By open joint surgeries or by total joint replacement, even disease in the late stage can be managed which leads to a good quality of life.

Financial or other competing interests: None.

Disclosure forms provided by the authors are available with the full text of this article at jemds.com.

\section{REFERENCES}

[1] Sodhi A, Naik S, Pai A, et al. Rheumatoid arthritis affecting temporomandibular joint. Contemp Clin Dent 2015;6(1):124-7.

[2] Helenius LMJ, Tervahartiala P, Helenius I, et al. Clinical, radiographic and MRI findings of the temporomandibular joint in patients with different rheumatic diseases. Int J Oral Maxillofac Surg 2006;35(11):983-9.

[3] Larheim TA, Smith HJ, Aspestrand F. Rheumatic disease of the temporomandibular joint: MR imaging and tomographic manifestations. Radiology 1990;175(2):527-31.

[4] Lin YC, Hsu ML, Yang JS, et al. Temporomandibular joint disorders in patients with rheumatoid arthritis. J Chin Med Assoc 2007;70(12):527-34.

[5] Crowson CS, Matteson EL, Myasoedova E, et al. The lifetime risk of adult-onset rheumatoid arthritis and other inflammatory autoimmune rheumatic diseases. Arthritis Rheum 2011;63(3):633-9.

[6] Gleissner C, Kaesser U, Dehne F, et al. Temporomandibular joint function in patients with longstanding rheumatoid arthritis - I. Role of periodontal status and prosthetic care - a clinical study. Eur J Med Res 2003;8(3):98-108.

[7] Gynther GW, Holmlund AB, Reinholt FP, et al. Temporomandibular joint involvement in generalized osteoarthritis and rheumatoid arthritis: a clinical, arthroscopic, histologic, and immunohistochemical study. Int J Oral Maxillofac Surg 1997;26(1):10-6.

[8] Moen K, Bertelsen LT, Hellem S, et al. Salivary gland and temporomandibular joint involvement in rheumatoid arthritis: relation to disease activity. Oral Dis 2005;11(1):27-34.

[9] Kallenberg A, Wenneberg B, Carlsson GE, et al. Reported symptoms from the masticatory system and general well-being in rheumatoid arthritis. J Oral Rehabil 1997;24(5):342-9.
[10] Seymour RL, Crouse VL, Irby WB. Temporomandibular ankylosis secondary to rheumatoid arthritis. Report of a case. Oral Surg Oral Med Oral Pathol 1975;40(5):584-9.

[11] Sidebottom AJ. Current thinking in temporomandibular joint management. $\mathrm{Br} \mathrm{J}$ Oral Maxillofac Surg 2009;47(2):91-4.

[12] Moore RA, Tramer MR, Carroll D, et al. Quantitative systematic review of topically applied non-steroidal anti-inflammatory drugs. BMJ 1998;316(7128):333-8.

[13] Lin J, Zhang W, Jones A, et al. Efficacy of topical nonsteroidal anti-inflammatory drugs in the treatment of osteoarthritis: meta-analysis of randomised controlled trials. BMJ 2004;329(7461):324.

[14] Cascos-Romero J, Vazquez-Delgado E, VazquezRodriguez E, et al. The use of tricyclic antidepressants in the treatment of temporomandibular joint disorders: systematic review of the literature of the last 20 years. Med Oral Patol Oral Cir Bucal 2009;14(1):E3-7.

[15] Al-Ani MZ, Davies SJ, Gray RJM, et al. Stabilisation splint therapy for temporomandibular pain dysfunction syndrome. Cochrane Database Syst Rev 2004;(1):CD002778.

[16] Aliko A, Ciancaglini R, Alushi A, et al. Temporomandibular joint involvement in rheumatoid arthritis, systemic lupus erythematosus and systemic sclerosis. Int J Oral Maxillofac Surg 2011;40(7):704-9.

[17] Bruce IN, Gladman DD. Psoriatic arthritis: recognition and management. BioDrugs 1998;9(4):271-8.

[18] Nell VPK, Machold KP, Eberl G, et al. Benefit of very early referral and very early therapy with disease-modifying anti-rheumatic drugs in patients with early rheumatoid arthritis. Rheumatology (Oxford) 2004;43(7):906-14.

[19] Sokka T, Mäkinen H. Drug management of early rheumatoid arthritis - 2008. Best Pract Res Clin Rheumatol 2009;23(1):93-102.

[20] Saad AA, Symmons DPM, Noyce PR, et al. Risks and benefits of tumor necrosis factor-alpha inhibitors in the management of psoriatic arthritis: systematic review and meta-analysis of randomized controlled trials. J Rheumatol 2008;35(5):883-90.

[21] Callhoff J, Sieper J, Weiß A, et al. Efficacy of TNFa blockers in patients with ankylosing spondylitis and non-radiographic axial spondyloarthritis: a metaanalysis. Ann Rheum Dis 2015;74(6):1241-8.

[22] Sidebottom AJ, Patel AA, Amin J. Botulinum injection for the management of myofascial pain in the masticatory muscles. A prospective outcome study. Br J Oral Maxillofac Surg 2013;51(3):199-205.

[23] Miloro M, Henriksen B. Discectomy as the primary surgical option for internal derangement of the temporomandibular joint. J Oral Maxillofac Surg 2010;68(4):782-9.

[24] Vasconcelos BC, Porto GG, Bessa-Nogueira RV. Condylar disk plication for temporomandibular joint internal derangement treatment: surgical technique and results. Med Oral Patol Oral Cir Bucal 2005;10 Suppl 2:E133-8.

[25] Nyberg J, Adell R, Svensson B. Temporomandibular joint discectomy for treatment of unilateral internal derangements--a 5 year follow-up evaluation. Int J Oral Maxillofac Surg 2004;33(1):8-12. 
[26] Helenius LMJ, Hallikainen D, Helenius I, et al. Clinical and radiographic findings of the temporomandibular joint in patients with various rheumatic diseases. A case-control study. Oral Surg Oral Med Oral Pathol Oral Radiol Endod 2005;99(4):455-63.

[27] Deoghare A, Degwekar SS. Clinical and CT scan evaluation of temporomandibular joints with osteoarthritis and rheumatoid arthritis. Journal of Indian academy of Oral Medicine and Radiology 2010;22(4):S15.
[28] Fathima N, Dharman S. Role of thermography in assessment of myogenous temperomandibular disorders. Biomed Pharmacol J 2019;12(3):1241-7.

[29] Kumaresan GD, Dhanraj M. Efficacy of Cox-2 inhibitors in the clinical management of TMJ arthritis: a review. Research J Pharm Tech 2017;10(12):4439-41.

[30] Marbach JJ. Arthritis of the temporomandibular joints. Am Fam Physician 1979;19(2):131-9.

[31] Zhu JF, Kaminski MJ, Pulitzer DR, et al. Psoriasis: pathophysiology and oral manifestations. Oral Dis 1996;2(2):135-44. 